\title{
An Internal Construction for Congruence Relations in Lattices
}

\author{
S G. Karpagavalli ${ }^{\text {a; }}$ and C. Ganesa Moorthy ${ }^{\mathrm{b}}$ \\ ${ }^{a}$ Department of Mathematics, Dr.Umayal Ramanathan college for women, Karaikudi-630 004, Tamil Nadu, \\ India. \\ ${ }^{b}$ Department of Mathematics, Alagappa University, Karaikudi-630 004, Tamil Nadu, India.
}

\begin{abstract}
A method of constructing a smallest congruence relation that is larger than a given equivalence relation on a lattice is explained. A method of constructing a congruence relation in which equivalence classes contain all least upper bounds and all greatest lower bounds for subsets of equivalence classes is explained; and this method constructs a smallest congruence relation with this property which is also larger than a given congruence relation in a lattice.
\end{abstract}

Keywords: Cardinal number, Transfinite induction principle, Congruence relation.

\section{Introduction}

Let $(\mathrm{L}, \leq)$ or $(\mathrm{L}, \vee, \wedge)$ be a lattice. Let $\aleph$ be an infinite cardinal number. A subset $\mathrm{A}$ of $\mathrm{L}$ is $\aleph$-closed in $\mathrm{L}$, if for any subset $\mathrm{B}$ of $\mathrm{A}$ for which $|\mathrm{B}|<\aleph$, the least upper bound of $\mathrm{B}$ is in $\mathrm{A}$, whenever it exists in $\mathrm{L}$, and the greatest lower bound of $B$ in $A$, whenever it exists in $\mathrm{L}$. A subset $\mathrm{A}$ of $\mathrm{L}$ is said to be closed in $\mathrm{L}$, if it $\aleph$ closed in $\mathrm{L}$, for every $\aleph$. An equivalence relation $\theta$ on $\mathrm{L}$ is said to be $\aleph$-closed, if every equivalence class induced by $\theta$ is $\aleph$-closed in $\mathrm{L}$. The equivalence relation $\theta$ on $\mathrm{L}$ is said to be closed, if every equivalence class induced by $\theta$ is closed. To an equivalence relation on $\mathrm{L}$, let us use an usual notation $\mathrm{x} \equiv \mathrm{y}(\bmod \theta)$, when $\mathrm{x}$ and $\mathrm{y}$ are related by $\theta$. Two equivalence relations $\theta$ and $\theta$ ' are ordered by the usual 'refinement' order relation: $\theta \leq \theta$ ' if $\mathrm{x} \equiv \mathrm{y}(\bmod \theta)$ implies $\mathrm{x} \equiv \mathrm{y}\left(\bmod \theta^{\prime}\right)$. An equivalence relation on $\mathrm{L}$ is said to be a congruence relation, if it has the following substitution properties: $\mathrm{x} \vee \mathrm{z} \equiv \mathrm{y} \vee \mathrm{z}(\bmod \theta)$ and $\mathrm{x} \wedge \mathrm{z} \equiv \mathrm{y} \wedge \mathrm{z}(\bmod \theta)$, whenever $\mathrm{x} \equiv \mathrm{y}(\bmod$ $\theta)$ and $x, y, z \in L$.

If $\left(\theta_{\mathrm{i}}\right)_{\mathrm{i} \in \mathrm{I}}$ is a collection of $\aleph$-closed (or, simply, closed) congruence relations on $\mathrm{L}$, then the relation $\theta$ on $\mathrm{L}$ defined by $\mathrm{x} \equiv \mathrm{y}(\bmod \theta)$ if and only if $\mathrm{x} \equiv \mathrm{y}\left(\bmod \theta_{\mathrm{i}}\right), \forall \mathrm{i} \in \mathrm{I}$, is also an $\aleph$-closed (or, simply, a closed) congruence relation on $\mathrm{L}$. Thus $\theta=\wedge_{i \in \mathrm{I}} \theta_{\mathrm{i}} \in \mathrm{\aleph} \mathrm{C}$ - ConL, the collection of all $\aleph$ - closed congruence relations on $\mathrm{L}$, when $\theta_{\mathrm{i}} \in \mathfrak{\aleph C}$ - ConL, $\forall \mathrm{i} \in \mathrm{I}$. Similarly $\vee_{\mathrm{i} \in \mathrm{I}} \theta_{\mathrm{i}}$ is in $\aleph \mathrm{C}$-ConL, when $\vee_{\mathrm{i} \in \mathrm{I}} \theta_{\mathrm{i}}$ is considered as $\wedge\{\phi$ $: \phi \in \mathrm{A}\}$, when $\mathrm{A}=\left\{\phi \in \mathrm{N} \mathrm{C}-\mathrm{C}\right.$ - $\left.\mathrm{L}: \theta_{\mathrm{i}} \leq \phi, \forall \mathrm{i} \in \mathrm{I}\right\}$. If ConL denotes the collection of all congruence relations on $\mathrm{L}$, it is known that ConL is a lattice, and an internal construction for least upper bound of a given sub collection is also known (see the proof of theorem 3.9 in [2]). So, an internal construction of $\vee_{i \in I} \theta_{i}$ in $\aleph C$ - ConL, when ( $\left.\theta_{\mathrm{i}}\right)_{\mathrm{i} \in \mathrm{I}} \subseteq \mathrm{K} \mathrm{C}$-ConL, depends on construction of a smallest $\theta$ " in $\aleph \mathrm{C}$-ConL such that $\theta$ " $\geq \theta$, for given $\theta \in \mathrm{ConL}$. For this construction, another internal constructon of a smallest congruence relation $\theta$ on $\mathrm{L}$ such that $\theta \geq \phi$ for a given equivalence relation $\phi$ on $\mathrm{L}$ is developed in this article. It is expected that all types of constructions may be helpful to understand congruence lattices(see: [3]).

\section{Construction of equivalence classes through transfinite induction}

At every phase, an equivalence relation is to be found from a given equivalence relation, by means of a construction. A finite set of constructions have to be repeated to reach a desirable equivalence relation. So, a common construction procedure is to be defined in this section.

Let $(L, \vee, \wedge)$ be a given lattice. To each $i=1,2, \ldots n$, a fixed positive integer, let $P_{i}($ say) denote a given common procedure which constructs an equivalence relation $\sim_{\mathfrak{i}}$ when it is applied on a given equivalence relation $\theta_{\mathrm{i}}$ with a result $\theta_{\mathrm{i}} \leq \sim_{\mathrm{i}}$. Let $\theta_{0}$ be a given equivalence relation on $\mathrm{L}$. Let $\theta_{\mathrm{i}}$ be the equivalence relation that is obtained by following the procedure $P_{i}$ on $\theta_{i-1}$, for $i=1,2, \ldots n$. For $m n<m n+i \leq m n+n$, let $\theta_{m n+i}$ denote the equivalence relation that is obtained by the following the procedure $P_{i}$ on $\theta_{m n+i-1}$, for $m=1,2 \ldots$. Now $\theta_{0} \leq \theta$ ${ }_{1} \leq \theta_{2} \leq \ldots$. Let $\theta_{\omega}$ be the supremum of the equivalence relations $\theta_{0}, \theta_{1}, \theta_{2,} \ldots$ in the lattice of all equivalence relations on L. Let us construct $\theta_{\omega+1} \theta_{\omega+2}, \ldots$ one by one by following the procedures $P_{1} ; P_{2} ; \ldots P_{n} ; P_{1} ; P_{2}, \ldots P_{n}, \ldots$ applied on $\theta_{\omega}, \theta_{\omega+1, \ldots}$ respectively. Now define $\theta_{\omega+\omega}$ as the supremum of the equivalence relations $\theta_{0}$, $\theta_{1}, \ldots, \theta_{\omega}, \theta_{\omega+1, \ldots}$ in the lattice of equivalence relations on L. Thus, once a limiting ordinal $\alpha$ is reached, $\theta_{\alpha}$ is constructed as the supremum of $\theta_{i}, i<\alpha$; and the procedures $P_{1}, P_{2}, \ldots P_{n}, P_{1}, P_{2}, \ldots P_{n} \ldots$ are applied on $\theta_{\alpha}, \theta_{\alpha+1}, \ldots$ to construct $\theta_{\alpha+1}, \theta_{\alpha+2}, \ldots$ respectively and successively. This procedure leads to a 'stationary' equivalence relation in the sense that $\theta_{\beta}=\theta_{\beta+\alpha}$, for every ordinal $\alpha$. This happens because $L$ is fixed and hence its cardinality 
is fixed. Let us say that the equivalence relation $\theta_{\beta}$ is the stationary equivalence relation obtained by following the procedures $P_{1}, P_{2}, \ldots P_{n}$ on $\theta_{0}$.

\section{Equivalence relation to congruence relation}

This section provides a construction to obtain a smallest congruence relation $\theta^{\prime}$ from a given equivalence relation $\theta$ such that $\theta \leq \theta^{\prime}$ on a lattice $\mathrm{L}$. This construction is based on the following significant observation.

Lemma 3.1 Let $\theta$ be a given equivalence relation on a lattice $(L, \vee, \wedge)$. To each $x \in L$, let $[x]$ denote the equivalence class of $\theta$ containing $\mathrm{x}$. Then $\theta$ is a congruence relation if and only if the following hold for every $\mathrm{x} \in \mathrm{L}$ : (i) $\mathrm{a} \vee \mathrm{b} \in[\mathrm{x}]$ and $\mathrm{a} \wedge \mathrm{b} \in[\mathrm{x}]$, whenever $\mathrm{a}, \mathrm{b} \in[\mathrm{x}]$; (ii) $(\mathrm{z} \vee \mathrm{a}) \wedge \mathrm{b} \in[\mathrm{x}]$ and $(\mathrm{z} \wedge \mathrm{a}) \vee \mathrm{b} \in[\mathrm{x}]$, whenever $\mathrm{a}, \mathrm{b} \in[\mathrm{x}]$, and $\mathrm{z} \in \mathrm{L}$; (iii) $\mathrm{a}_{1} \vee \mathrm{b}_{1} \in[\mathrm{x}]$, whenever $\mathrm{a} \vee \mathrm{b} \in[\mathrm{x}], \mathrm{a}_{1} \in[\mathrm{a}]$ and $\mathrm{b}_{1} \in[\mathrm{b}]$; (iv) $\mathrm{a}_{1} \wedge \mathrm{b}_{1} \in[\mathrm{x}]$, whenever $\mathrm{a} \wedge \mathrm{b} \in[\mathrm{x}], \mathrm{a}_{1} \in[\mathrm{a}]$ and $\mathrm{b}_{1} \in[\mathrm{b}]$.

Proof: The proof follows from two facts:

(1) $\theta$ is a congruence relation if and only if $L / \theta$ is a lattice.

(2) A set with two binary operations is a lattice if and only if the binary operations satisfy idempotent law, commutativity law, associativity law, and absorption law (see: Theorem 1 in p.18 in [1]).

Suppose (i),(ii),(iii) and (iv) are true. To each $a, b \in L$, let us define $[a] \vee[b]=[a \vee b]$

and $[a] \wedge[b]=[a \wedge b]$. They are well defined in view of (iii) and (iv). The commutavity and associativity of these operations follow from the corresponding properties of $\vee$ and $\wedge$ in $L$. These operations satisfy idempotent law and absorption law, because of (i) and (ii). So L/ $\theta$ is a lattice so that $\theta$ is a congruence relation.

On the other hand, if $\theta$ is a congruence relation, then $\mathrm{L} / \theta$ is a lattice so that (i), (ii),(iii) and (iv) are true.

Construction procedure $\mathbf{P}_{1}$ : Let $\theta$ be a given equivalence relation on a lattice $(L, \vee, \wedge)$. Define $\mathrm{x} \sim_{1} \mathrm{y}$ if there is a finite sequence $\mathrm{x}_{1}, \mathrm{y}_{1}, \mathrm{x}_{1}{ }^{\prime}, \mathrm{y}_{1}{ }^{\prime}, \mathrm{x}_{2}, \mathrm{y}_{2}, \mathrm{x}_{2}{ }^{\prime}, \mathrm{y}_{2}, \ldots \mathrm{x}_{\mathrm{n}}, \mathrm{y}_{\mathrm{n}}, \mathrm{x}_{\mathrm{n}}{ }^{\prime}, \mathrm{y}_{\mathrm{n}}, \ldots .$. in $\mathrm{L}$ such that $\mathrm{x}_{\mathrm{i}}{ }^{\prime} \equiv \mathrm{x}_{\mathrm{i}}(\bmod \theta)$ and $\mathrm{y}_{\mathrm{i}}{ }^{\prime} \equiv \mathrm{y}_{\mathrm{i}}(\bmod \theta)$, $\forall \mathrm{i}=1,2, \ldots \mathrm{n}$, and such that $\mathrm{x}=\mathrm{x}_{1} \wedge \mathrm{y}_{1}, \mathrm{y}=\mathrm{x}_{\mathrm{n}}{ }^{\prime} \wedge \mathrm{y}_{\mathrm{n}}{ }^{\prime}, \mathrm{x}_{\mathrm{i}}{ }^{\prime} \wedge \mathrm{y}_{\mathrm{i}}{ }^{\prime}{ }^{\prime}=\mathrm{x}_{\mathrm{i}+1}{ }^{\prime} \wedge \mathrm{y}_{\mathrm{i}+1}{ }^{\prime}, \forall \mathrm{i}=1,2, \ldots \mathrm{n}-1$. Then $\sim_{1}$ is an equivalence relation on L. Let us say that $\sim_{1}$ is obtained from $\theta$ by following procedure $\mathrm{P}_{1}$. Observe that if $\mathrm{x}_{1} \equiv \mathrm{x}_{2}(\bmod \theta)$ and $\mathrm{y}_{1} \equiv \mathrm{y}_{2}(\bmod \theta)$, then $\mathrm{x}_{1} \wedge \mathrm{y}_{1} \sim \sim_{1} \mathrm{x}_{1} \wedge \mathrm{y}_{2}$. Note that $\theta \leq \sim_{1}$.

Construction procedure $\mathbf{P}_{2}$ : Replace $\sim_{1}, \mathrm{P}_{1}, \wedge$ in the previous discussion by $\sim_{2}, \mathrm{P}_{2}, \vee$, respectively, so that if $\mathrm{x}_{1} \equiv \mathrm{x}_{2}(\bmod \theta)$ and $\mathrm{y}_{1} \equiv \mathrm{y}_{2}(\bmod \theta)$, then $\mathrm{x}_{1} \wedge \mathrm{y}_{1} \sim \sim_{2} \mathrm{x}_{2} \vee \mathrm{y}_{2}$. Note that $\theta \leq \sim_{2}$.

Construction procedure P3: To each $\mathrm{x} \in \mathrm{L}$, let $[\mathrm{x}]$ denote the equivalence class of a given equivalence relation $\theta$ on a given lattice $(L, \vee, \wedge)$. Let us define a relation $\sim_{3}$ on $L$ by $a \sim_{3} b$ if there is a finite sequence $a_{0}, a_{1}, a_{2}, \ldots, a_{n}$ in L such that : (i) $\mathrm{a} \equiv \mathrm{a}_{0}(\bmod \theta)$; (ii) $\mathrm{b} \equiv \mathrm{a}_{\mathrm{n}}(\bmod \theta)$; and (iii) to each $\mathrm{i}=0,1,2, \ldots, \mathrm{n}-1$, there $\mathrm{are}_{\mathrm{i}}, \mathrm{c}_{\mathrm{i}} \in\left[\mathrm{a}_{\mathrm{i}}\right]$ such that $b_{i} \vee c_{i} \in\left[a_{i+1}\right]$ or $b_{i} \wedge c_{i} \in\left[a_{i+1}\right]$; or there are $b_{i+1}, c_{i+1} \in\left[a_{i+1}\right]$ such that $b_{i+1} \vee c_{i+1} \in\left[a_{i}\right]$ or $b_{i+1} \wedge c_{i+1} \in\left[a_{i}\right]$. Then $\sim_{3}$ is an equivalence relation. Let us say that ' $\sim_{3}$ ' is obtained from $\theta$ by following procedure $\mathrm{P}_{3}$. Observe that if $\mathrm{b}_{0}, \mathrm{c}_{0}$ $\in\left[\mathrm{a}_{0}\right]$, then $\mathrm{a}_{0} \sim \sim_{3} \mathrm{~b}_{0} \vee \mathrm{c}_{0}$ and $\mathrm{a}_{0} \sim{ }_{3} \mathrm{~b}_{0} \wedge \mathrm{c}_{0}$. Note that $\theta \leq \sim_{3}$.

Construction procedure $\mathbf{P}_{4}$ : Let us fix $\mathrm{L}$ and $\theta$, and let us fix the notation $[\mathrm{x}]$ as in the previous procedure. Let us define a relation $\sim \sim_{4}$ on $\mathrm{L}$ by $\mathrm{a} \sim \sim_{4} \mathrm{~b}$ if there is a finite sequence $\mathrm{a}_{0}, \mathrm{a}_{1}, \ldots, \mathrm{a}_{\mathrm{n}}$ in $\mathrm{L}$ such that: (i) $\mathrm{a} \equiv \mathrm{a}_{0}(\bmod \theta)$; (ii) $\mathrm{b} \equiv \mathrm{a}_{\mathrm{n}}(\bmod \theta)$; and (iii) to each $\mathrm{i}=1,2, \ldots, \mathrm{n}-1$ there are $\mathrm{b}_{\mathrm{i}}, \mathrm{c}_{\mathrm{i}} \in\left[\mathrm{a}_{\mathrm{i}}\right]$ and $\mathrm{di} \in \mathrm{L}$ such that $\left(\mathrm{d}_{\mathrm{i}} \wedge \mathrm{b}_{\mathrm{i}}\right) \vee c i \in\left[\mathrm{a}_{\mathrm{i}+1}\right]$ or $\left(\mathrm{d}_{\mathrm{i}} \vee \mathrm{b}_{\mathrm{i}}\right) \wedge \mathrm{c}_{\mathrm{i}} \in\left[\mathrm{a}_{\mathrm{i}+1}\right]$; or there are $\mathrm{b}_{\mathrm{i}+1}, \mathrm{c}_{\mathrm{i}+1} \in\left[\mathrm{a}_{\mathrm{i}+1}\right]$ and $\mathrm{d}_{\mathrm{i}+1} \in \mathrm{L}$ such that $\left(\mathrm{d}_{\mathrm{i}+1} \wedge \mathrm{b}_{\mathrm{i}+1}\right) \vee \mathrm{c}_{\mathrm{i}+1} \in\left[\mathrm{a}_{\mathrm{i}}\right]$ or $\left(\mathrm{d}_{\mathrm{i}+1} \vee \mathrm{b}_{\mathrm{i}+1}\right) \wedge \mathrm{c}_{\mathrm{i}+1} \in\left[\mathrm{a}_{\mathrm{i}}\right]$. Then ' $\sim$ ' ' is an equivalence relation. Let us say that ' $\sim$ ' ' is obtained from $\theta$ by following procedure $\mathrm{P}_{4}$. Observe that if $\mathrm{b}_{0}, \mathrm{c}_{0} \in\left[\mathrm{a}_{0}\right]$ and $\mathrm{d}_{0} \in \mathrm{L}$, then $\mathrm{a}_{0} \sim \sim_{4}\left(\mathrm{~d}_{0} \vee \mathrm{b}_{0}\right) \wedge \mathrm{c}_{0}$ and $\mathrm{a}_{0} \sim 4\left(\mathrm{~d}_{0} \wedge \mathrm{b}_{0}\right) \vee \mathrm{c}_{0}$. Note that $\theta \leq \sim_{4}$.

Theorem 3.2 Let $\theta_{0}$ be a given equivalence relation on a lattice $(L, \vee, \wedge)$. Let $\theta_{\beta}$ be the stationary equivalence relation obtained by following the procedures $\mathrm{P}_{1}, \mathrm{P}_{2}, \mathrm{P}_{3}, \mathrm{P}_{4}$ on $\theta_{0}$. Then $\theta_{\beta}$ is the smallest congruence relation on $\mathrm{L}$ such that $\theta_{0} \leq \theta_{\beta}$. .Proof: To each $\mathrm{x} \in \mathrm{L}$, let $[\mathrm{x}]$ denote the equivalence class of $\theta_{\beta}$ containing $\mathrm{x}$. The procedure $\mathrm{P}_{1}, \mathrm{P}_{2}, \mathrm{P}_{3}$, and $\mathrm{P}_{4}$ reveal that the conditions (i),(ii),(iii) and (iv) of the previous lemma 3.1 are satisfied for the equivalence relation $\theta_{\beta}$ on $L$. So, $\theta_{\beta}$ is a congruence relation on $L$ such that $\theta_{0 \leq} \theta_{\beta}$.

\section{Equivalence relation to closed congruence relation}

This section provides a construction to obtain a smallest closed equivalence relation $\theta$ ' from a given equivalence relation $\theta$ such that $\theta \leq \theta$ ' on a lattice. This construction may be combined with the construction of the previous section to obtain a smallest closed congruence relation. Let us introduce some notations for the next construction. Let $\theta$ be an equivalence relation in a given lattice $\mathrm{L}$. If $\mathrm{K}_{1}, \mathrm{~K}_{2}$ are subsets of two equivalence classes of $\theta$, then let us write $K_{1} \equiv K_{2}(\bmod \theta)$ if $K_{1}, K_{2}$ are subsets of the same equivalence class of $\theta$, then let us 
write $\mathrm{x} \equiv \mathrm{K}(\bmod \theta)$ if $\mathrm{x} \equiv \mathrm{y}(\bmod \theta), \forall \mathrm{y} \in \mathrm{K}$. If $\mathrm{K}$ is a subset of $\mathrm{L}$ and if a least upper bound of $\mathrm{K}$ exists in $\mathrm{L}$, then it will be denoted by $\vee \mathrm{K}$. The notation $\wedge \mathrm{K}$ refer to a greatest lower bound on $\mathrm{K}$, when it exists.

Construction procedure $\mathbf{P}_{5}$ : Let $\theta$ be a given equivalence relation on a lattice $(L, \vee, \wedge)$. Let $\aleph$ be a fixed infinite cardinal number. Let us write $\mathrm{H} \sim \mathrm{K}$ for two non empty subsets $\mathrm{H}, \mathrm{K}$ of equivalence classes of $\theta$, when there is a finite sequence $\mathrm{G}_{0}, \mathrm{G}_{1}, \ldots, \mathrm{G}_{\mathrm{n}}(\mathrm{n} \geq 1)$ of subsets of equivalence classes of $\theta$ such that:

(i) $\mathrm{H} \equiv \mathrm{G}_{0}(\bmod \theta)$,

(ii) $\mathrm{K} \equiv \mathrm{G}_{\mathrm{n}}(\bmod \theta)$,

(iii) $\left|\mathrm{G}_{\mathrm{i}}\right|<\aleph, \forall \mathrm{i}=1,2, \ldots, \mathrm{n}$

(iv) $\vee \mathrm{G}_{\mathrm{i}}$ exists and $\vee \mathrm{G}_{\mathrm{i}}=\mathrm{G}_{\mathrm{i}+1}(\bmod \theta)$ or $\wedge \mathrm{G}_{\mathrm{i}}$ exists and $\wedge \mathrm{G}_{\mathrm{i}}=\mathrm{G}_{\mathrm{i}+1}(\bmod \theta)$ or

$\vee G_{i+1}$ exists and $\vee G_{i+1} \equiv G_{i}(\bmod \theta)$ or

$\wedge \mathrm{G}_{\mathrm{i}+1}$ exists and $\wedge \mathrm{G}_{\mathrm{i}+1}=\mathrm{G}_{\mathrm{i}}(\bmod \theta)$, for $\mathrm{i}=1,2, \ldots, \mathrm{n}-1$.

The following can be verified.

(i) If $\mathrm{x} \equiv \mathrm{y}(\bmod \theta)$, then $[\mathrm{x}] \sim[\mathrm{y}]$. For take $\mathrm{n}=1, \mathrm{G}_{0}=\mathrm{H}=\{\mathrm{x}\}$ and $\mathrm{G}_{1}=\mathrm{K}=\{\mathrm{y}\}$ in the previous description.

(ii) Let $\mathrm{H}$ be a subset of an equivalence class of $\theta$ such that $|\mathrm{H}|<\aleph$.

If $\vee \mathrm{H}$ exists, then $\mathrm{H} \sim\{\vee \mathrm{H}\}$. For, take $n=1, \mathrm{G}_{0}=\mathrm{H}$, and $\mathrm{G}_{1}=\mathrm{K}=\{\vee \mathrm{H}\}$ in the previous description. Similarly, if $\wedge \mathrm{H}$ exists, then $\mathrm{H} \sim\{\wedge \mathrm{H}\}$.

Let us now define ' $\sim_{5}$ ' on $\mathrm{L}$ by $\mathrm{x} \sim_{5} \mathrm{y}$ if $[\mathrm{x}] \sim[\mathrm{y}]$, when $\mathrm{x}, \mathrm{y} \in \mathrm{L}$. This defines an equivalence relation on $\mathrm{L}$ such that $\theta \leq \sim_{5}$. Let us say that ' $\sim_{5}$ ' is obtained from $\theta$ by following the procedure $\mathrm{P}_{5}$. The properties (i) and (ii) of ' $\sim 5$ ' imply the next theorem 4.1 .

Theorem 4.1 Let $\theta_{0}$ be a given equivalence relation on a lattice $(L, \vee, \wedge)$. Let $\aleph$ be a given infinite cardinal number. Let $\theta_{\beta}$ be the stationary equivalence relation obtained by following the procedure $P_{5}$ on $\theta_{0}$. Then $\theta_{\beta}$ is the smallest $\aleph$-closed equivalence relation on $\mathrm{L}$ such that $\theta_{0} \leq \theta_{\beta}$.

The next theorem 4.2 is a combination of Theorem 3.2 and Theorem 4.1 .

Theorem 4.2 Let $\theta_{0},(L, \vee, \wedge)$ and $\aleph$ be as in the previous theorem 4.1. Let $\theta_{\beta}$ be the stationary equivalence relation obtained by following the procedures $\mathrm{P}_{1}, \mathrm{P}_{2}, \mathrm{P}_{3}, \mathrm{P}_{4}, \mathrm{P}_{5}$ on $\theta_{0}$. Then $\theta_{\beta}$ is the smallest $\aleph$-closed congruence relation on $\mathrm{L}$ such that $\theta_{0} \leq \theta_{\beta}$.

Remark 4.3 If $\left(\theta_{\mathrm{i}}\right)_{\mathrm{i} \in \mathrm{I}}$ is a collection of equivalence relations on a lattice $(\mathrm{L}, \vee, \wedge)$, then one can follow an usual procedure (see theorem 4.3 in p.23 in [1]) to construct a smallest equivalence relation $\theta_{0}$ such that $\theta_{\mathrm{i}} \leq \theta_{0}, \forall \mathrm{i} \in \mathrm{I}$ If $\theta_{\beta}$ is the congruence relation constructed in the theorem 4.2 , then $\theta_{\beta}$ is the smallest $\aleph$-closed congruence relation such that $\theta_{\mathrm{i}} \leq \theta_{\beta}, \mathrm{i} \in \mathrm{I}$.

Remark 4.4 The word ' $\aleph$-closed' may be replaced by the word 'closed' in the discussion of this section.

\section{References}

[1] G.Birkoff, Lattice Theory, Amer. Math. Soc., New York, 1948.

[2] T.S.Blyth, Lattices and ordered structures, Springer, London, 2005.

[3] F.Wehrung, A solution to Dilworth's congruence lattice problem, Advances in Mathematics, 216(2007)610-625. 